\title{
Spectral Energy Distributions of LIRGs
}

\author{
Vivian $\mathbf{U}^{1,2,3}$, D. B. Sanders ${ }^{3}$, and the GOALS Team ${ }^{4}$ \\ ${ }^{1}$ NASA Jenkins Predoctoral Fellow \\ ${ }^{2}$ SAO Predoctoral Fellow, Harvard-Smithsonian CfA, Cambridge, MA 02138, USA \\ ${ }^{3}$ Institute for Astronomy, University of Hawaii, Honolulu, HI 96822, USA \\ ${ }^{4}$ http://goals.ipac.caltech.edu \\ Email: vivian@ifa.hawaii.edu
}

\begin{abstract}
We present preliminary results from a study of the SEDs of a complete sample of 65 LIRGs from GOALS. The spectral shapes at $\lambda>10 \mu \mathrm{m}$ are similar, while the largest variations occur in the $\mathrm{NIR}\left(L_{1 \mu \mathrm{m}}^{5 \mu \mathrm{m}} / L_{\odot} \sim 1.0-0.01\right)$ and $\mathrm{UV}\left(L_{1 \mu \mathrm{m}}^{0.12 \mu \mathrm{m}} / L_{\odot} \sim 2.0-0.005\right)$. Using stellar population synthesis models to fit the UV-NIR continuum data, we derive stellar masses for the host galaxies of $\log \left(M_{\star} / M_{\odot}\right) \sim 10.2-11.4$ with a mean of $\sim 10.8$.
\end{abstract}

Keywords. galaxies: interactions; galaxies: fundamental parameters

Luminous infrared galaxies (LIRGs) in the local universe are predominantly galaxy mergers and represent an important transition stage in galaxy evolution (e.g. Sanders \& Mirabel 1996). In order to characterize their physical properties and better understand the nature of their internal energy sources, we are carrying out a detailed study of the spectral energy distributions (SEDs) of a complete sub-sample of 65 local LIRGs with $\log \left(L_{\mathrm{IR}} / L_{\odot}\right)=11.0-12.5\left(H_{0}=70 \mathrm{~km} \mathrm{~s}^{-1} \mathrm{Mpc}^{-1}, \Omega_{\mathrm{m}}=0.28, \Omega_{\Lambda}=0.73\right)$ from the Great Observatories All-sky LIRG Survey (GOALS: Armus et al. 2009). By using a single mask for each object to determine the total magnitudes from UV through radio, we are better able to assess the variations in their SEDs (see Figure 1) and to derive total stellar masses. A more complete analysis will be presented by $\mathrm{U}$ et al. (in preparation).

\section{References}

Armus, L., et al. 2009, PASP, 30, 490

Sanders, D. B. \& Mirabel, I. F. 1996, ARAA, 34, 749

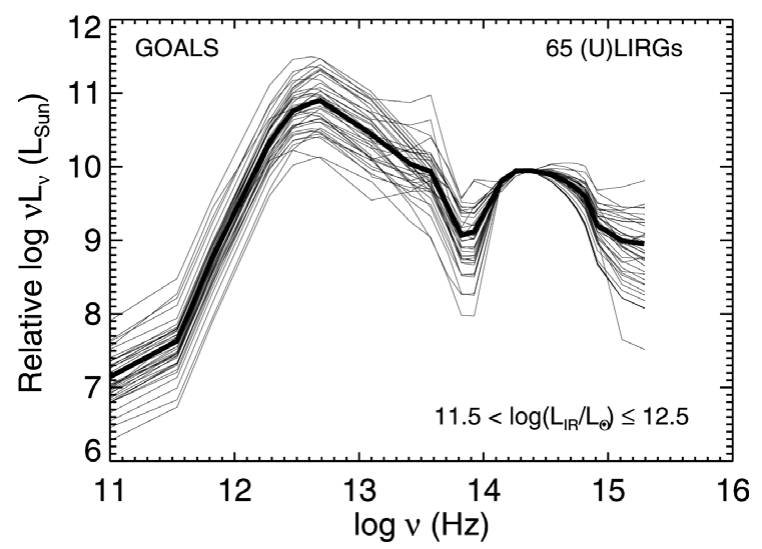

Figure 1. FUV-submm $(0.12-850 \mu \mathrm{m})$ SEDs of a complete sub-sample of local LIRGs with $\log \left(L_{\mathrm{IR}} / L_{\odot}\right)=11.0-12.5$, normalized at $J$-band. 